\title{
Untersuchung über den Wasserwechsel der Seeigel und seine Bedeutung für Atmung und Exkretion*
}

\author{
H. Fechter \\ Abteilung für Wirbellose Tiere, Zoologische Staatssammlung; München, \\ und \\ Biologische Anstalt Helgoland (Meeresstation); Helgoland, \\ Bundesrepublik Deutschland
}

\begin{abstract}
Studies on water exchange of sea urchins and its importance for respiration and excretion. Measurements of the quantities of sea water taken in by the mouth and expelled by the anus show that Echinus esculentus L. drinks water at more or less regular intervals. The amounts of water taken in through the mouth increase allometrically in relation to body volume. Upon stimulation, drinking rate may rise to more than ten times the basic rate. Since the amount of water leaving the body through the anus exceeds that entering through the mouth, additional water must be absorbed through the body surface and excreted by the gut. The amount of water influx via body surface is especially high in smaller individuals. Drinking, influx and excretion of water are discussed with regard to respiration and excretion: hasty drinking, caused by stimulation, can increase the supply of oxygen to the perivisceral cavity, and subsequent excretion supports the removal of metabolic wastes. The following mechanism of water influx is proposed and discussed: As a consequence of excess $\mathrm{K}^{+}$and $\mathrm{Ca}^{++}$ions in the ambulacral system, due to an ion pump, there exists a small inward directed osmotic gradient causing water to enter the lumina of the expanded tube feet. When the tube feet contract, most of the absorbed water is forced through the ampulla walls into the perivisceral cavity, from where it is actively excreted by the gut, together with metabolic wastes.
\end{abstract}

\section{EINLEITUNG}

An den allermeisten Stoffwechselvorgängen ist Wasser als Lösungsmittel, Reaktionsmilieu, Reaktionspartner oder 'Transportmittel entscheidend und unentbehrlich beteiligt. Als Vehikel der Stoffwechselprodukte durchfließt es mit diesen den Tierkörper und verläßt ihn, mehr oder weniger stark mit seinen Stoffwechselendprodukten angereichert, über die Ausscheidungsorgane. Das durch die Ausscheidung der wasserlöslichen Stoffwechselendprodukte dem Körper entzogene Wasser muß wieder ergänzt werden. Im allgemeinen geschieht dies durch Aufnahme wasserreicher Nahrung, im besonderen durch Trinken. Nur selten treten im Tierreich Formen auf, die ihren Wasserbedarf fast ausschließlich durch Oxidation aus extrem trockener Nahrung gewinnen

* Mit Unterstützung der Deutschen Forschungsgemeinschaft. 
(z. B. Mehl- und Kleidermotte). Während die Landtiere außer dem durch Ausscheidung verlorengegangenen Wasser auch noch die Verdunstungsverluste ergänzen müssen, haben die im Wasser lebenden Tiere, je nach dem osmotischen Gradienten zwischen ihrem Innen- und dem Außenmedium, gegen zuviel eindringendes Wasser - hypertones Innenmedium bei Süßwasser- und Brackwassertieren sowie euryhalinen Meerestieren oder gegen Austrocknung - hypotones Innenmedium bei den marinen Teleosteern und einigen Krebsen - zu kämpfen.

Wie aber liegen die Verhältnisse bei isotonen Meerestieren, die sich weder einer Überschwemmung ihres Innenmediums mit Wasser noch einer Auszehrung desselben wegen osmotischer Differenzen zwischen Innen und Außen zu erwehren haben? Wie die meisten anderen Tiere scheiden sie wasserlösliche Stoff wechselendprodukte aus, was mit der Ausscheidung von unterschiedlich großen Wassermengen einhergeht. Wie ergänzen sie nun diesen Wasserverlust? Durch Wasseraufnahme über die durchlässige Körperoberfläche, durch Trinken oder auf beiden Wegen?

Abgesehen von diesen allgemeinen Fragen bieten die isotonen, zur Osmoregulation unfähigen Echinodermen ein spezielles, mit dem Wasserwechsel eng verknüpftes Problem. Sie besitzen keines der im Tierreich sonst verbreiteten Exkretionsorgane (Nieren) und es ist nach wie vor unklar, auf welchem Wege ihre löslichen Stoffwechselendprodukte den Körper verlassen. $\mathrm{Da}$ die Echinodermen mit ihrem isotonen Innenmedium nur Wasserverluste durch die exkretorische Tätigkeit erleiden, ergibt sich hier die Möglichkeit über den Weg des Wasserwechsels den Ort der Ausscheidung zu ermitteln.

\section{MATERIAL UND METHODE}

Als Versuchsobjekt wurde Ecbinus esculentus L. gewählt, der leicht in genügender Anzahl zu erhalten ist und eine ausreichende Körpergröße und Schalenfestigkeit bietet, die das Anbringen der Versuchsapparatur gestattet. Diese bestand aus zwei sechseckigen Plexiglasplatten (Abb. 1), von denen die eine auf sechs mit Gewinde versehenen V2A-Stahlstäben gegen die andere verschiebbar war. Sie dienten zum Anpressen zweier zylindrischer, einseitig offener Plexiglaskammern (A, B) an die Oral- bzw. Aboralseite der Seeigelschale. Der freie Rand der offenen Kammerseite war jeweils von einem Schaumgummiring (SR) eingesäumt, der beim Anpressen an die Schalenoberfläche eine ausreichende Dichtungsfläche ergab. Der Durchmesser der Kammern wurde so gewählt, daß diese möglichst eng um das Peristom bzw. Periproct anlagen und der größte Teil der Schalenoberfläche frei blieb. Jede Kammer war mit zwei Rohrstutzen $\left(a, a^{*} ; b, b^{*}\right)$ zum Anschluß von Silikonschläuchen versehen. Die Schläuche an $b$ und $b^{*}$ dienten zum Absaugen eingeschlossener Luftblasen nach dem Anbringen der Kammern am Seeigel, jene an a und $a^{*}$ führten $z u$ den Meßgefäßen für die Wasseraufnahme durch den Mund (C) und die Wasserabgabe durch den After (D).

Das schwierigste Problem bestand darin, im Trinkgefäß $(C)$ stets einen genügend großen Wasservorrat für die Wasseraufnahme zur Verfügung zu haben, ohne dadurch einen Druckunterschied zum Auffangbehälter für das ausgeschiedene Wasser zu erzeugen. Dies wurde durch die Verwendung eines nach dem Prinzip der Mariotteschen Flasche gebauten, graduierten Vorratsgefäßes (C) vermieden, indem über ein langes 
Rohr (E), das tief in das Gefäß hineinragte, der in der freien Atmosphäre herrschende Druck unter den Wasserspiegel des Vorratsgefäßes, genau in die Höhe des Wasserspiegels im Versuchsbecken (WS) gelegt wurde. Befindet sich die Mündung der Zuleitung (F) zum Auffanggefäß (D) ebenfalls im Niveau des Versuchsbeckenwasser-

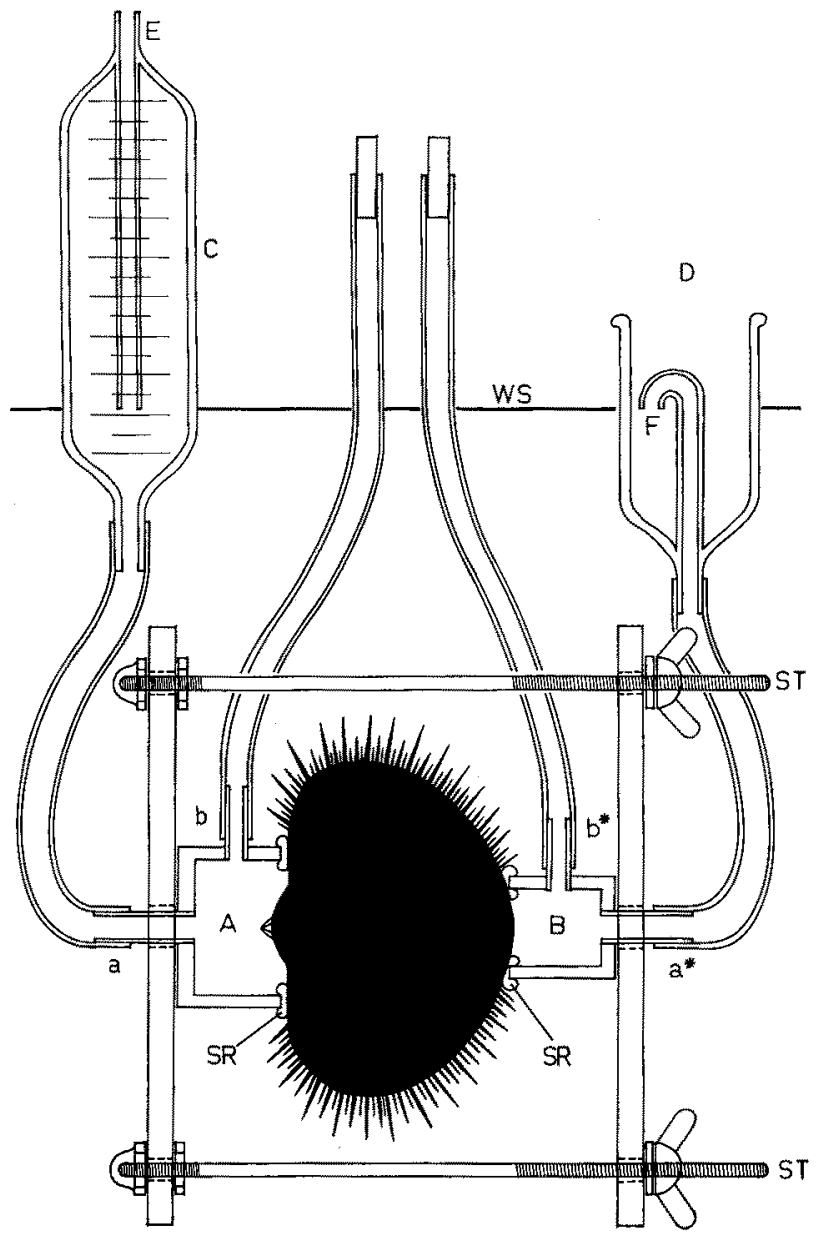

Abb. 1: Versuchsanordnung (Erklärung im Text)

spiegels, so herrscht in den Kammern A und B derselbe Druck wie er im Mittel auch auf der Seeigeloberfläche lastet. Damit sind im gesamten Versuchssystem ausgeglichene Druckverhältnisse geschaffen, die denen in der natürlichen Umwelt des Versuchsobjektes entsprechen.

Zum wasserdichten Aufsetzen der Kammern auf die Schalenoberfläche mußten von dieser rund um das Mundfeld und den After alle Stacheln, unter möglichst geringer Verletzung, sorgfältig nacheinander abpräpariert werden. Danach wurde den 
Seeigeln in einem gesonderten Becken, das gut durchströmt und belüftet war, bei täglicher mehrmaliger Reinigung durch Abbrausen, Gelegenheit zur Regeneration der Epithelverletzungen gegeben. Nach der Ausheilung kamen die Tiere in die Versuchsapparatur, in der sie zwischen den zwei Kammern A und B und den beiden sechseckigen Platten durch Anziehen der Flïgelschrauben so festgeklemmt wurden, daß die Schaumgummiringe (SR) wasserdicht auf die Schalenoberfläche gepreßt waren, jedoch kein übermäßig großer Druck auf die Schale ausgeübt wurde. An Perlonfäden hängend, die an einem der Stahlstäbe (ST) befestigt waren, wurde das ganze in ein gut belïftetes und durchströmtes Becken gesenkt und die beiden, mit filtriertem Seewasser gefüllten und mit vollen Leitungsschläuchen versehenen Meßgefäße (C, D) in einem gemeinsamen Halter in der für ausgeglichene Druckverhältnisse notwendigen Lage am Wasserspiegel des Beckens einjustiert. Nach dem Offnen der Verbindung (E) zur freien Atmosphäre am Trinkgefäß (C) wurden die Leitungsschläuche mit den Rohrstutzen der Kammern (A, B) verbunden und das im Auffanggefäß (D) befindliche Wasser abgesaugt. Die Versuche mit einem Tier erstreckten sich jeweils über 10-20 Stunden.

Insgesamt präparierte ich 10 Seeigel für die Versuche. 5 davon bekamen jedoch Infektionen mit Blaualgen an den Wundstellen und konnten für die Versuche nicht mehr verwendet werden. Von den restlichen 5 wurde jedes Tier nach einigen Tagen einem Kontrollversuch unterworfen.

Die aus dem Trinkgefäß aufgenommene Wassermenge wurde anhand der vorher geeichten Graduierung desselben gemessen, die in das Auffanggefäß abgegebene Menge mit Hilfe einer Meßpipette ermittelt. Die Bestimmung des Körpervolumens - genauer gesagt des von der Seeigelschale umschlossenen Raumes - erfolgte durch Auswiegen mit Wasser. Hierzu wurden die Eingeweide entfernt, die Schale wurde getrocknet, auf der Außenseite mit Wachs versiegelt, vor sowie nach dem Auffüllen mit destilliertem Wasser gewogen und der absolute Betrag der Gewichtsdifferenz dem Körpervolumen gleichgesetzt.

\section{ERGEBNISSE}

\section{Die theoretisch möglichen Wege des Wasserwechsels}

Als Eintrittspforte für die Wasseraufnahme kommt in erster Linie die Mundöffnung in Frage, durch die Wasser einmal zusammen mit der Nahrung, zum anderen außerhalb der Freßzeiten durch Trinken einverleibt werden kann. Das über die Mundöffnung aufgenommene Wasser kann den Darm, Nebendarm oder beide gemeinsam passieren und unverändert oder mit Exkretstoffen beladen und sauerstoffärmer durch den After wieder austreten. Es kann aber auch ganz oder teilweise im Vorder- und Mitteldarm resorbiert werden, die Leibeshöhlen und Organe durchströmen und über den Enddarm, die Körperoberfläche oder durch beide zugleich den Körper wieder verlassen und auf diesem Wege Stoffwechselendprodukte mit nach außen transportieren.

Neben der Mundöffnung bietet die gesamte ausgedehnte Körperoberfläche mit ihren Differenzierungen Möglichkeiten zum Wassereintritt durch die Füßchen und, in bescheidenerem Umfang, sicher auch durch die Schale selbst. Das über die Körper- 


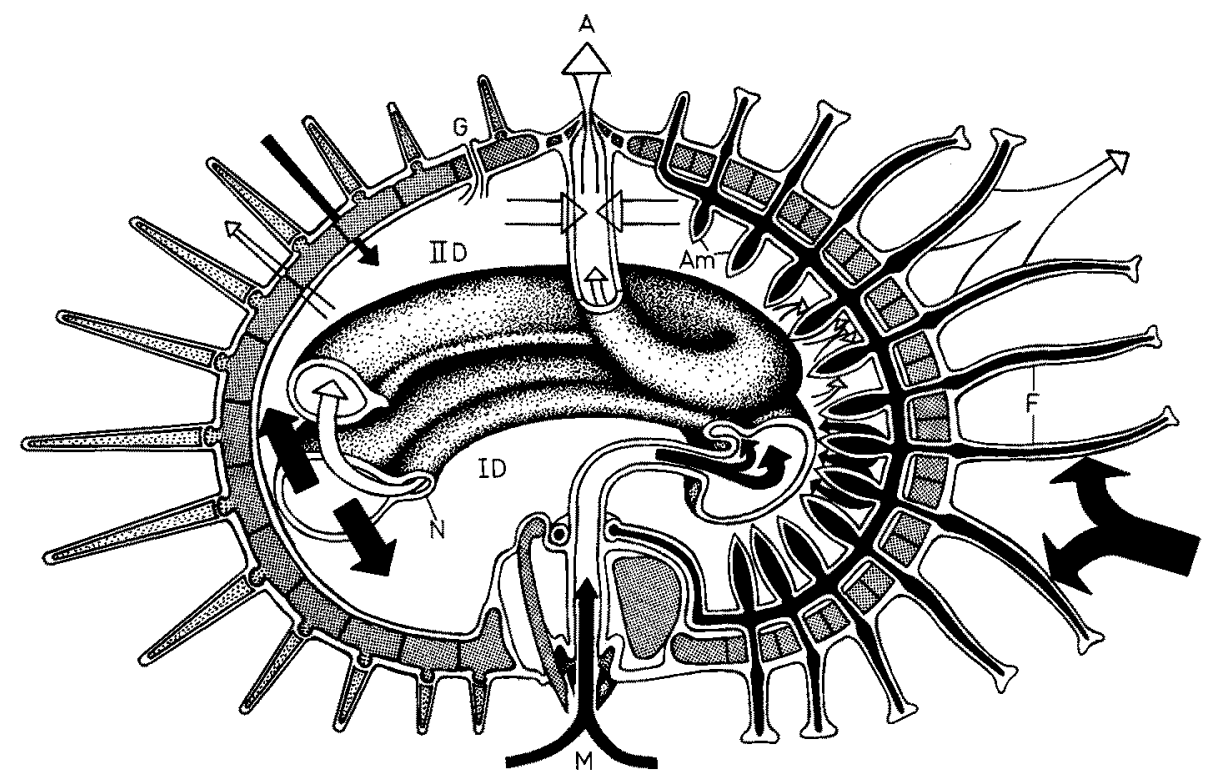

(1) Mund $\longrightarrow$ Darm $<\begin{aligned} & \text { direkte Passage durch Darm } \longrightarrow \text { und / oder Nebendarm } \\ & \text { Leibeshöhlen+Organe }<\text { Körperoberfläche }\end{aligned}$

(2) Körperoberfläche $\longrightarrow$ Leibeshöhlen + Organe $<$ Körperoberfläche

Abb. 2: Theoretisch mögliche Wege des Wasserwechsels. Die schwarzen Pfeile kennzeichnen in den Körper eintretende, die weißen den Körper verlassende Wasserströme. A After; $A m$ Ampullen; I D 1. Darmschlinge; II D 2. Darmschlinge; $F$ Füßschen; $G$ Geschlechtsöffnung; $M$ Mund; $N$ Nebendarm

oberfläche eingedrungene Wasser durchsetzt Leibeshöhlen und Organe und kann durch den Darm, die Körperoberfläche oder beide wieder ausgeschieden werden.

Ein weiterer Weg des Wasserwechsels wäre theoretisch auch noch über das System Steinkanal-Axialorgan--Madreporenplatte denkbar. Doch konnte bereits durch frühere Arbeiten (KRÜGER 1932, FEchTER 1965) nachgewiesen werden, daß auf diesem Wege weder ein Wasserein- noch ein Wasserausstrom erfolgt. Zusammenfassend lassen sich also zwei Wasserwege feststellen, die in Abbildung 2 schematisch dargestellt sind.

Die Versuchsapparatur gestattete die Messung der durch den Mund aufgenommenen und den After abgegebenen Wassermenge. Die über die Körperoberfläche eingedrungene bzw. ausgeschiedene Menge ist nicht direkt meßbar. Sie läßt sich aber in zwei von drei möglichen fällen indirekt erfassen. 
Stellt man eine Bilanz des Wasserwechsels durch den Mund und After auf, so ergeben sich drei Möglichkeiten: (1) Die durch den Mund aufgenommene Menge $W_{m}=$ der durch den After abgegebenen $W_{a}$. (2) Die durch den Mund aufgenommene Menge ist größer als die durch den After abgegebene. (3) Die durch den Mund aufgenommene Menge ist kleiner als die durch den After abgegebene.

Im ersten Falle $W_{m}=W_{a}$ lassen sich aus den Meßergebnissen keine Schlußfolgerungen hinsichtlich des tatsächlich vorliegenden Weges des Wasserwechsels ziehen, da es immerhin denkbar ist, daß sich ein über die Körperoberfläche eindringender und wieder durch sie austretender Wasserstrom die Waage halten oder ein Teil des durch den Mund aufgenommenen Wassers resorbiert und über die Körperoberfläche $a b$ gegeben, dafür aber ein gleich großer, über die Körperoberfläche eindringender Teil durch den Darm ausgeschieden wird. Im Falle $W_{m}>W_{a}$ muß die Differenz $W_{m}-W_{a}$ durch die Körperoberfläche nach außen gegangen und im Falle $W_{m}<W_{a}$ die Menge $W_{a}-W_{m}$ über diese in den Körper eingedrungen sein.

Das im Seeigelkörper selbst durch Oxidation von Nährstoffen gebildete Wasser läßt sich in seiner maximal überhaupt möglichen Menge aus der Sauerstoffaufnahme berechnen. Das meiste Wasser, unter geringstem $\mathrm{O}_{2}$-Verbrauch, entsteht bei der Oxidation von Glucose. Pro ${ }^{1 / 6} \mathrm{~mol}$ Glucose und dem Verbrauch von $1 \mathrm{~mol} \mathrm{O} 2$ entsteht $1 \mathrm{~mol} \mathrm{H}_{2} \mathrm{O}$, d. h. pro $\mathrm{ml} \mathrm{O}_{2}$ werden $0,8035 \mu \mathrm{I} \mathrm{H}_{2} \mathrm{O}$ erzeugt.

Nun sind leider für die Echinoiden nur sehr spärliche Daten über die Sauerstoffaufnahme und den Verbrauch vorhanden und die wenigen bekannten Werte differieren außerordentlich stark. So geben JoHANSEN \& VADAs (1967) für Strongylocentrotus purpuratus (STrmpson) $8 \mu 1 \mathrm{O}_{2}$ pro Gramm-Naßgewicht und Stunde an, während Giese et al. (1966) für dieselbe Art 26-31 $\mu \mathrm{l} \mathrm{O}_{2} / \mathrm{g} / \mathrm{h}$ und Farmanfarmaian (1966) $36 \mu 1 \mathrm{O}_{2} / \mathrm{g} / \mathrm{h}$ ermittelt haben. Koller \& MEYER (1933) fanden für Psammechinus miliaris (P. L. S. MülLER) gar den außergewöhnlich hoch erscheinenden Wert von 57...78 $\mu \mathrm{l} \mathrm{O} / \mathrm{g} / \mathrm{h}$. STEEN (1965) kam bei seinen Untersuchungen an Strongylocentrotus droebachiensis (O. F. MÜLLER), der in seinen Ausmaßen am ehesten mit Echinus esculentus $\mathrm{L}$. vergleichbar ist, maximal auf $28 \mu \mathrm{O} \mathrm{O} / \mathrm{g} / \mathrm{h}$. Uber Echinus esculentus $\mathrm{L}$. selbst sind bedauerlicherweise keine Angaben vorhanden, doch dürfte der entsprechende Wert um $30 \mu \mathrm{O}_{2} / \mathrm{g} / \mathrm{h}$ liegen. Um jedoch den theoretisch möglichen Maximalbetrag an Oxidationswasser zu erhalten, muß mit dem größten bisher bekannten, in der Extrapolation aber sicherlich zu hohen Wert von $78 \mu \mathrm{l} \mathrm{O} / \mathrm{g} / \mathrm{h}$ gerechnet werden, um den Einfluß dieser Wasserquelle auf die Bilanz des Wasserwechsels abschätzen zu können. Danach könnte ein $150 \mathrm{~g}$ schwerer E. esculentus in 10 Stunden maximal $0,094 \mathrm{ml} \mathrm{H}_{2} \mathrm{O}$ durch Veratmen von Glucose erzeugen. Diese Wassermenge ist in Hinblick auf den Gesamtumfang des Wasserwechsels zu vernachlässigen.

\section{Wasseraufnahme durch Trinken}

Die graphische Darstellung (Abb. 3) der Wasseraufnahme durch Trinken über einen Zeitraum von 20 Stunden zeigt, daß größere Tiere mehr als kleine trinken und die getrunkene Menge zeitproportional ist. Dabei fällt auf, daß in den ersten Stunden nach Versuchsbeginn die Trinkraten (Tab. 2) erheblich größer sind als etwa im Zeit- 
raum zwischen der 3. und 20. Stunde, in dem sie konstante Werte annehmen. Diese erhöhten Trinkraten zu Beginn sind mit Sicherheit auf eine gesteigerte Erregung der Tiere durch die ungewohnten Versuchsbedingungen zurückzuführen. Die Erregung ist an den zunächst heftigeren Stachelbewegungen, dem Stachelsperren, und den ruhelosen Suchbewegungen der Ambulacralfüßchen erkennbar. Sie klingt im Verlauf der ersten Stunden immer mehr ab, bis schließlich ein Ruhezustand erreicht wird, der von dem eines freien, am Boden festgehefteten Seeigels äußerlich nicht zu unterscheiden ist.

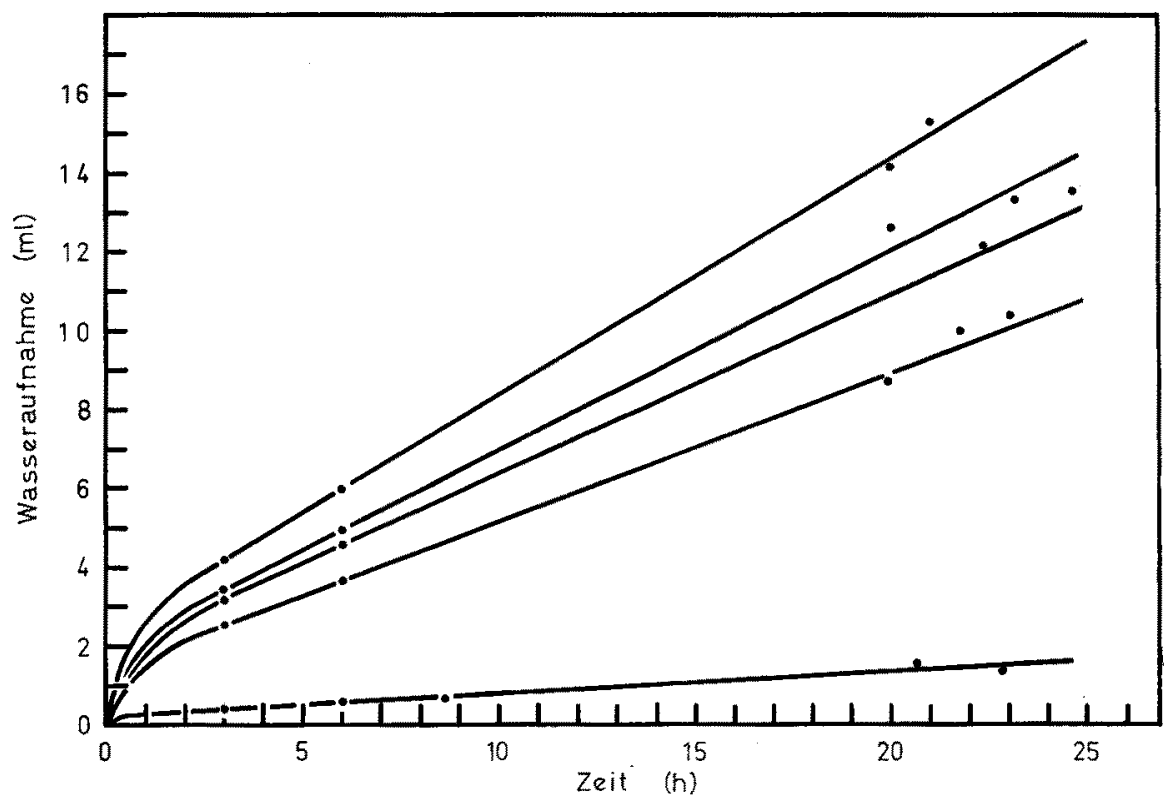

Abb. 3: Zeitlicher Verlauf der Wasseraufnahme durch Trinken bei fünf unterschiedlich großen Versuchstieren

Daß äußere Reize eine Steigerung der Trinkrate bewirken, konnte in einem Versuch gezeigt werden, bei dem die Seeigel in einigen Zeitabständen durch Berühren und Bewegen der Stacheln immer wieder mechanisch gereizt wurden, was jedesmal heftiges Trinken auslöste (Abb. 4). Ein Seeigel konnte auf diese Weise dazu gebracht werden, in einem Zeitraum von einer Stunde $10,76 \mathrm{ml}$ zu trinken, eine Menge, die unter normalen Versuchsbedingungen von einem Seeigel vergleichbarer Größe erst in etwa 18 Stunden bewältigt wird.

Beim Trinken sind nur gelegentlich Bewegungen des Zahnapparates erkennbar. Das Offnen und Schließen des Mundes erfolgt somit unabhängig von Kieferbewegungen.

Stellt man die Trinkraten (Tab. 1) in Abhängigkeit vom Körpervolumen in einem doppeltlogarithmischen Koordinatensystem dar, so liegen die Werte annähernd auf einer Geraden (Abb. 5). Eine Ausnahme davon machen allerdings die Werte für den Seeigel mit dem kleinsten Volumen. Sie liegen weit $a b$ und sind vergleichsweise außer- 


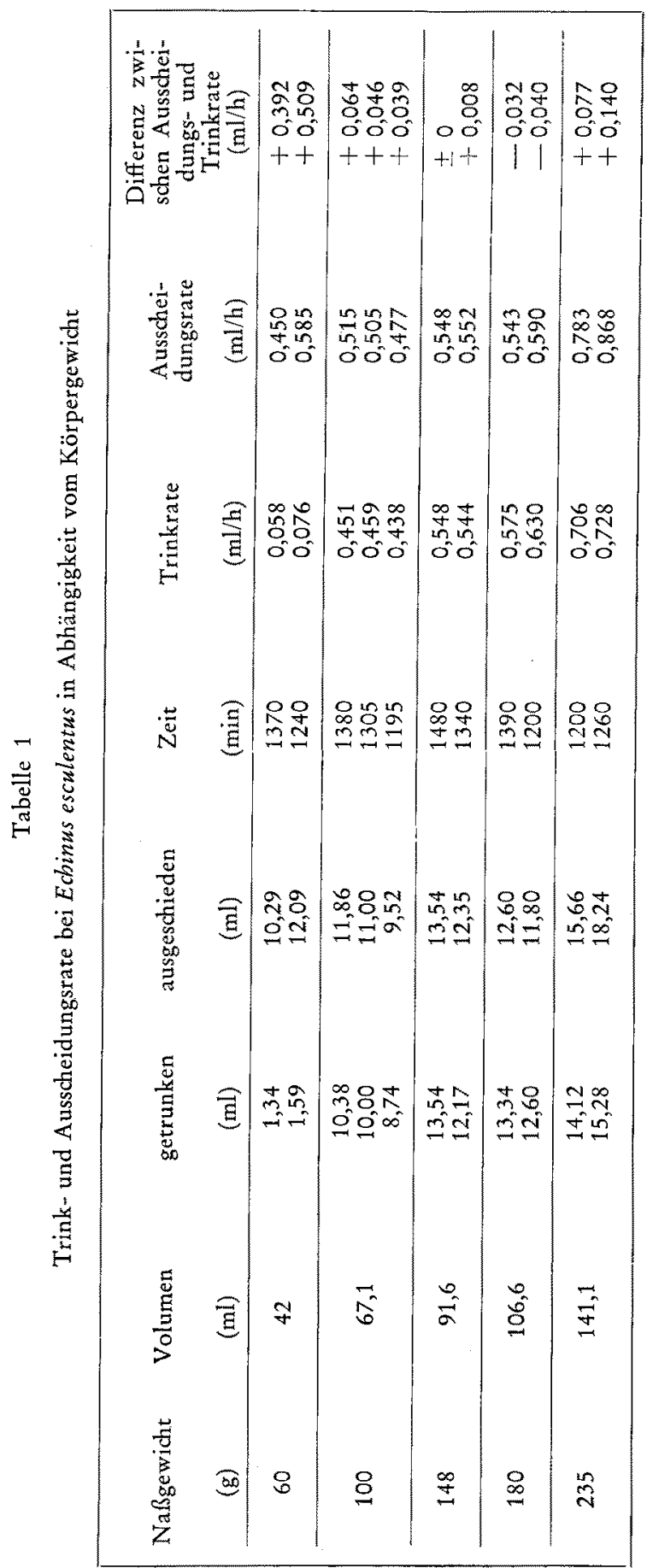




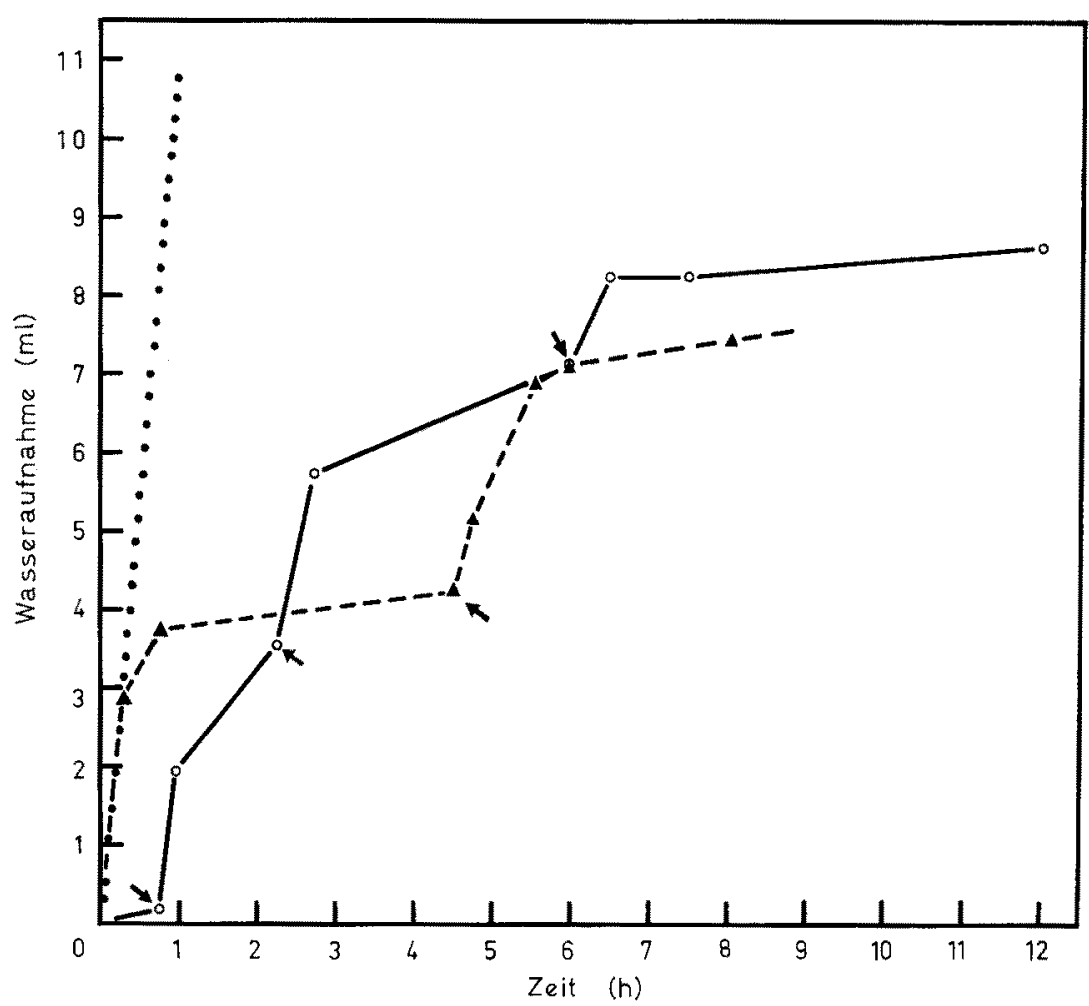

Abb. 4; Zeitlicher Verlauf des Trinkens bei mechanischer Reizung der Stacheln. Die Pfeile zeigen das Einsetzen der Reize an. Dauerreizung ergab den gepunkteten Verlauf

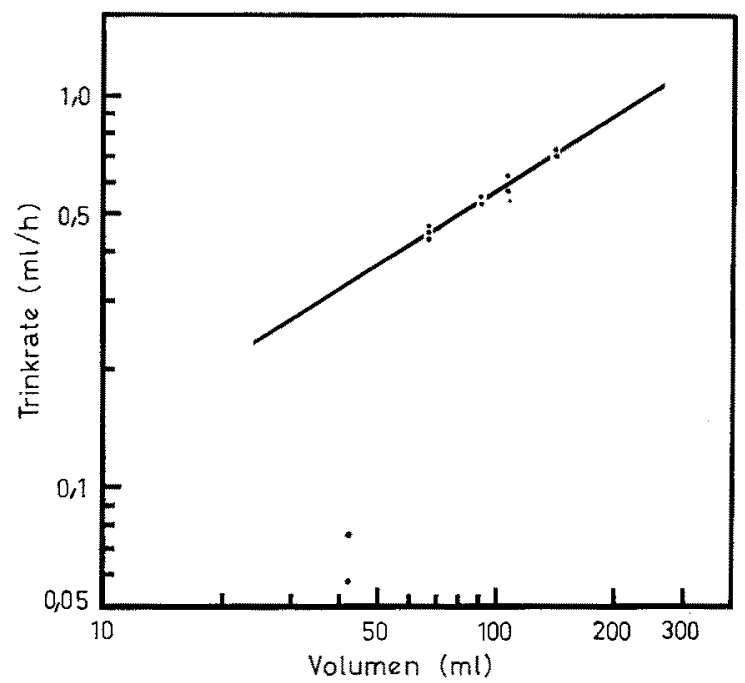

Abb. 5. Abhängigkeit der Trinkrate vom Körpervolumen 
ordentlich niedrig. Dies legt die Vermutung nahe, daß kleine Seeigel entweder sehr selten trinken oder bei jedem Schluck nur eine relativ geringe Wassermenge aufnehmen.

Nach der Methode der kleinsten Quadrate ergibt sich bei Ausklammerung der Werte für den kleinsten Seeigel aus der Neigung der Regressionsgeraden für die allometrische Beziehung $W_{t r}=a V^{b}$ zwischen Trinkrate $\left(W_{t r}\right)$ und Körpervolumen $(V)$ ein Exponent $(b)$ von 0,63 .

Dieses Ergebnis wird durch folgende Uberlegungen bestätigt: Für die Aufnahme und Passage der getrunkenen Wassermenge sind bestimmte Voraussetzungen hinsichtlich des Querschnittes der Mundöffnung und des Pharynx gegeben. Je größer der

Tabelle 2

Mittelwerte der Trinkraten $(\mathrm{ml} / \mathrm{h})$ bei Echinus esculentus

\begin{tabular}{ccc|}
\hline $\begin{array}{c}\text { Volumen } \\
(\mathrm{ml})\end{array}$ & $\begin{array}{c}\text { Trinkrate innerhalb } \\
\text { der ersten 3 Stunden }\end{array}$ & $\begin{array}{c}\text { Trinkrate zwischen der } \\
\text { 3. und 20. Stunde }\end{array}$ \\
\hline 42 & 0,133 & 0,055 \\
67,1 & 0,850 & 0,379 \\
91,6 & 1,07 & 0,454 \\
106,6 & 1,15 & 0,506 \\
141,1 & 1,40 & 0,597 \\
\hline
\end{tabular}

Querschnitt, desto größer auch die bei jedem Schluck einverleibte Wassermenge. Mit anderen Worten, die pro Schluck getrunkene Wassermenge $W_{s}$ ist dem wirksamen Querschnitt $Q$ der Mundöffnung proportional.

$$
W_{s}=k Q
$$

Ist $d$ der Durchmesser der Mundöffnung, so wird

$$
\mathrm{Q}=\frac{1}{4} \pi d^{2} \text { und } W_{s}=\frac{1}{4} k \pi d^{2}
$$

Es kann angenommen werden, daß der Durchmesser der Mundöffnung mit wachsendem Körpervolumen im gleichen Maße zunimmt wie der Pharynx-Durchmesser.

Tabelle 3

Beziehung zwischen Pharynxdurchmesser und Körpervolumen

\begin{tabular}{|cc|}
\hline $\begin{array}{c}\text { Volumen } \\
(\mathrm{ml})\end{array}$ & $\begin{array}{c}\text { Pharynxdurchmesser } \\
(\mathrm{cm})\end{array}$ \\
\hline 11,24 & 0,127 \\
25,11 & 0,127 \\
25,28 & 0,191 \\
28,44 & 0,191 \\
27,71 & 0,159 \\
36,47 & 0,222 \\
49,53 & 0,254 \\
72,39 & 0,222 \\
102,0 & 0,254 \\
167,3 & 0,318 \\
249,2 & 0,382 \\
317,0 & 0,318 \\
\hline
\end{tabular}




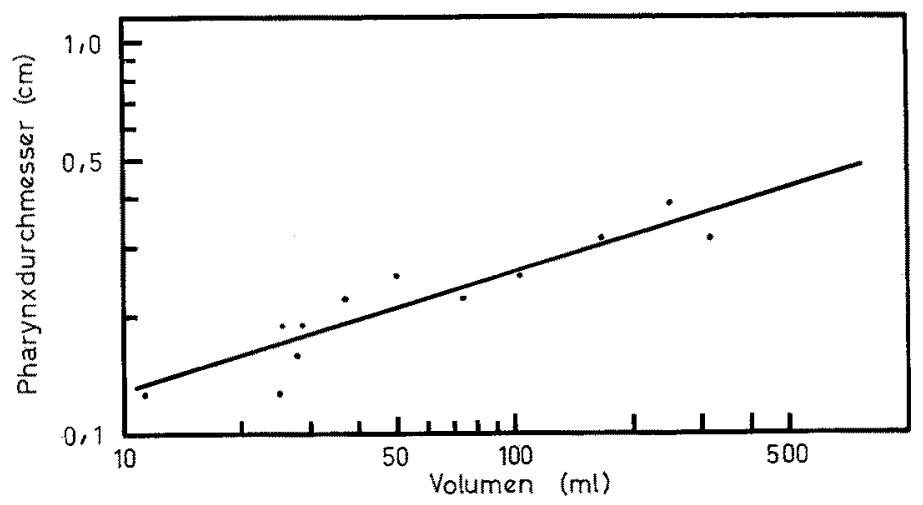

Abb. 6: Beziehung zwischen Pharynx-Durchmesser und Körpervolumen

In Tabelle 3 sind die Durchmesser des Pharynx - gemessen unmittelbar über dem Kiefergerüst - von 12 Individuen von Echinus esculentus wiedergegeben. Die Darstellung (Abb. 6) der Wertepaare im doppeltlogarithmischen Koordinatensystem zeigt, daß zwischen dem Pharynxdurchmesser $d$ und dem Körpervolumen $V$ eine allometrische Beziehung besteht, für die die Regressionsrechnung einen Exponenten von 0,31 ergibt.

$$
d=a_{1} V^{0,31}
$$

In (1) eingesetzt, erhält man die pro Schluck getrunkene Wassermenge

$$
W_{s}=\frac{1}{4} k \pi a_{1}^{2} V^{0,62}
$$

Die Trinkrate $W_{t r}$ ergibt sich aus der Trinkfrequenz $f$, d. h. der Anzahl der Schlucke pro Zeiteinheit und der pro Schluck aufgenommenen Wassermenge $W_{s}$

$$
W_{t r}=f W_{s}=\frac{1}{4} f k \pi a_{1}^{2} V^{0,62}
$$

Nimmt man an, daß die Trinkfrequenz vom Körpervolumen unabhängig konstant bleibt und setzt man

so wird (2)

$$
\frac{1}{4} f k \pi a_{1}^{2}=a
$$

$$
W_{t r}=a V^{0,62}
$$

Der Exponent entspricht fast genau der tatsächlich gefundenen Neigung der Regressionsgeraden in der Beziehung $z$ wischen der Trinkrate und dem Körpervolumen.

\section{Wasserabgabe durch den After}

Versuchsanordnung und Meßgenauigkeit gestatteten es leider nicht, die Wasserabgabe durch den After, so wie dies beim Trinken möglich war, kontinuierlich quantitativ zu verfolgen. Es wurde jeweils nur die gesamte, bis zum Ende des Versuches 
ausgeschiedene Menge gemessen. Die Versuchsdauer währte im einzelnen unterschiedlich lange, betrug aber in jedem Falle mindestens 20 Stunden (vgl. Tab. 1).

Die Versuchsanordnung erlaubte es jedoch zu beobachten, wann und wie häufig Flüssigkeit durch den After ausgestoßen und wann und wie oft getrunken wurde. Demnach erfolgen Trinken und Ausscheiden selten gleichzeitig. Weder die Wasseraufnahme noch die Wasserabgabe finden in streng regelmäßigen Intervallen statt oder folgen einander in bestimmtem zeitlichem Abstand. Die einzige erkennbare Regelhaftigkeit war, daß längere, gelegentlich $1 / 2$ bis 1 Stunde andauernde Ruhepausen mit Perioden gehäufter Trink- bzw. Ausscheidungsaktivität abwechselten, in denen in rasch aufeinanderfolgenden Zügen getrunken oder in mehreren Schüben Flüssigkeit ausgestoßen wurde, worauf wieder Ruhepausen eintraten.

Mechanische Reizung steigert nicht nur die Trinkfrequenz, sondern auch die Ausscheidungsfrequenz. Auf den Initialreiz reagiert der Seeigel meist mit gleichzeitigem Trinken und Ausscheiden. Man hat den Eindruck, daß es sich dabei um eine Art von Schreckreaktion handelt. Nachfolgende Reize bewirken zunächst überwiegend eine Steigerung des Trinkens. Erst nach einer Reihe von Schlucken wird dann mit einem kräftigen Schub Wasser ausgestoßen. Den Wasserausstoß beim Initialreiz bewirkt wahrscheinlich nicht allein die Kontraktion der Darmmuskulatur, er ist teilweise sicher auch auf die aus der Schreckreaktion sich ergebende Füßchenkontraktion und das Einziehen des Kieferapparates zurückzuführen, die eine Flüssigkeitsverdrängung aus dem Darmsystem verursachen.

Eine erneute Schreckreaktion ist erst nach einer längeren Pause in der Reizeinwirkung zu erzielen. Unmittelbar auf den ersten Reiz folgende Reize haben keine Kontraktion der Füßchen bzw, der Kiefermuskulatur mehr zur Folge, sondern wirken im Gegenteil eher stimulierend auf die Füßchenbewegung; man hat den Eindruck, als wollte der Seeigel fliehen. Daher dürfte der unter den nachfolgenden Reizen auftretende Wasserausstoß allein die Folge der erhöhten Wasseraufnahme sein.

Die im Versuchszeitraum durch den After ausgeschiedenen Flüssigkeitsmengen sind in Tabelle 1 wiedergegeben. In Beziehung zum Körpervolumen nimmt die Ausscheidungsrate zunächst nur langsam zu und steigt erst ab einer bestimmten Körpergröße stärker an.

\section{Bilanz des Wasserwechsels}

Vergleicht man die durch Trinken aufgenommene Wassermenge mit der durch den After ausgeschiedenen und bildet die Differenz, so ergibt sich, daß in 8 von 11 Fällen (72,7\%) mehr Flüssigkeit ausgeschieden als getrunken wurde (vgl. Tabelle 1). Nur in einem Fall ist gleich viel getrunken und ausgeschieden, in zwei Fällen weniger ausgeschieden als getrunken worden.

Dabei fällt besonders auf, daß vor allem der kleinste Seeigel, der am wenigsten getrunken hat, die größte zusätzliche Flïssigkeitsmenge ausschied. In den darauffolgenden drei Größenklassen nimmt diese zusätzlich ausgeschiedene Menge ständig $a b$, und erst bei dem voluminösesten der hier untersuchten Seeigel steigt sie erneut an.

Inwieweit die Werte bei der verhältnismäßig kleinen Zahl von Versuchstieren repräsentativ sein mögen, sei dahingestellt. Das starke Uberwiegen der Fälle jedoch, 


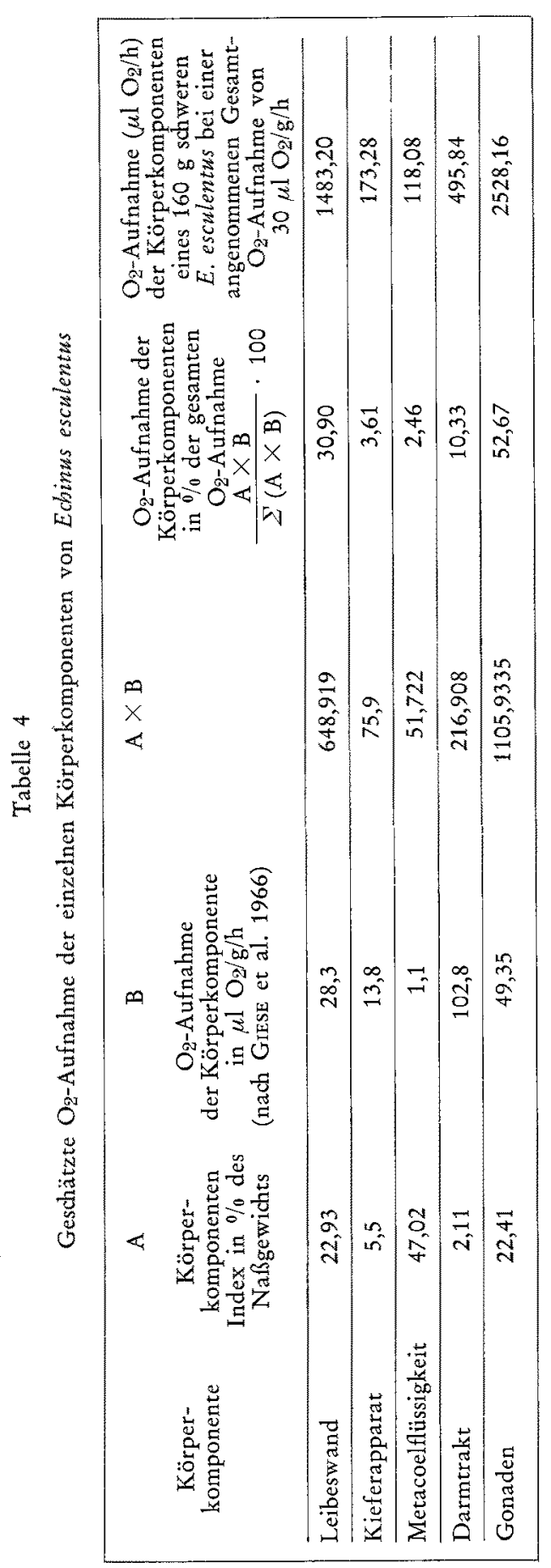


in denen zusätzliche Flüssigkeitsmengen ausgeschieden wurden, berechtigt zu dem Schluß, daß bei Ecbinus esculentus nicht unerhebliche Wassermengen über die Körperoberfläche in das Metacoel eindringen und durch den Darm wieder ausgeschieden werden.

\section{DISKUSSION UND SCHLUSSFOLGERUNGEN}

Wie die Versuchsergebnisse zeigen, trinken Seeigel, zwar portionsweise, aber ziemlich kontinuierlich. Sie steigern die Trinkrate bei Erregung und scheiden in der Regel mehr aus, als sie trinkend aufnehmen. Das in den Körper eintretende Wasser bringt Sauerstoff mit, das den Körper verlassende Wasser kann Stoffwechselendprodukte nach außen transportieren. Es erscheint deshalb angebracht, die Ergebnisse im Hinblick auf ihre Bedeutung für Atmung und Exkretion zu diskutieren.

\section{Bedeutung für die Atmung}

Wie Farmanfarmatan (1966) am Beispiel von Strongylocentrotus purpuratus gezeigt hat, können Gewebeschichten aus $\mathrm{O}_{2}$-gesättigtem Meerwasser auf dem Diffusionswege nur bis zu einer Tiefe von $1,5 \mathrm{~mm}$ mit Sauerstoff versorgt werden. Daraus folgt, daß bei Seeigeln nur Füßchen- und Darmwand genügend dünn sind, um ausreichend $\mathrm{O}_{2}$ hindurchdiffundieren zu lassen. Farmanfarmaian (1966) und Steen (1965) demonstrierten auch auf eindrucksvolle Weise, daß die Sauerstoffzufuhr zum Körperinneren fast ausschließlich über die Füßchen und deren Ampullen stattfindet.

Dies konnte durch eigene Versuche, bei denen die als Redoxindikator dienende Leukoform des Methylenblau in das Metacoel eingespritzt wurde, für Echinus esculentus bestätigt werden. Der farblose Redoxindikator färbt sich in Gegenwart von Sauerstoff blau. Die Sektion der Versuchstiere in verschiedenen Zeitabständen nach der Injektion ergab, daß sich unmittelbar nach der Injektion alle Ampullen des Wassergefüßsystems intensiv blau färben, während das Coelothel des Interradialbereiches erst nach etwa 30 Minuten schwach blau gefärbt war, wobei es sich vermutlich um einen reinen Färbungsprozeß von seiten der inzwischen blau gewordenen Coelomflüssigkeit handelte.

Wiederholt wurde, zuerst von Perrier (1875), zuletzt von Stott (1955), die Vermutung ausgesprochen, daß der Nebendarm bzw. die Siphonalrinne der Seeigel nicht nur dazu dient, Wasser, das die Verdauungssäfte im Magen zu verdünnen droht, an diesem vorbeizuleiten, sondern vor allem auch dazu, der 2. Darmschlinge Atemwasser zuzuführen, von wo der Sauerstoff entweder direkt über die Darmwand in die angrenzende Metacoelflüssigkeit (Perivisceralflüssigkeit) diffundiert oder in die darmumspinnenden Blutbahnen gelangt und über das Gefäßssystem verbreitet wird.

Der Sauerstoffpartialdruck $\mathrm{pO}_{2}$ in der Metacoelflüssigkeit beträgt nach FredericQ (1911) bei Sphaerechinus granularis (LAM.) 0,027-0,059 atm, bei Paracentrotus lividus (LAm.) 0,026 atm, was im Mittel $(0,037)$ den Werten STEENs entspricht, der bei Strongylocentrotus droebacbiensis (O. F. MüLLER) 0,03-0,04 atm gefunden hat. Der $\mathrm{pO}_{2}$ der Metacoelflüssigkeit ist also stets wesentlich geringer als der des umgebenden 
$\mathrm{O}_{2}$-gesättigten Meerwassers (0,21 atm). Vom $\mathrm{O}_{2}$-gesättigten Meerwasser im Darm zur Metacoelflüssigkeit besteht daher ein beträchtlicher Druckgradient. Bisher war nicht bekannt, ob dem Darm überhaupt Wasser zugeführt wird und, falls dies zutriff, in welchem Ausmaß es geschieht. Wie aus Tabelle $1 \mathrm{zu}$ ersehen ist, beträgt die Trinkrate eines in Ruhe befindlichen E. esculentus von $180 \mathrm{~g}$ etwa $0,5 \mathrm{ml} / \mathrm{h} . \mathrm{O}_{2}$-gesättigtes Meerwasser von $35 \%$ Salzgehalt enthält bei $15^{\circ} \mathrm{C}$ rund $6 \mu \mathrm{O} \mathrm{O} / \mathrm{ml}$. Bei vollständiger Resorption des Sauerstoffes kann der Seeigel also einen zusätzlichen Sauerstoffgewinn von $3 \mu \mathrm{l} \mathrm{O} / \mathrm{h}$ erzielen.

Farmanfarmaian fand bei Srongylocentrotus purpuratus, denen er die Darmpassage verschloß, eine Verminderung der Sauerstoffaufnahme um $0,1 \mu \mathrm{l} \mathrm{O} 2 / \mathrm{min}$; das sind $6 \mu \mathrm{O}_{2} / \mathrm{h}$, was der vollständigen Resorption des Sauerstoffs aus einer Trinkrate

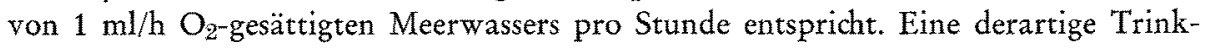
rate besitzt ein E. esculentus mit einem Körpervolumen von etwa $90 \mathrm{ml}$ in den ersten drei Stunden nach Versuchsbeginn (vgl. Tab. 2) in einer Phase abklingender Erregung. Da man annehmen darf, daß sich auch FARMAnFARMAIANs Versuchstiere in einer solchen Phase befanden und zudem die Körpergröße des zitierten $E$. esculentus mit der eines großen Exemplares von Strongylocentrotus purpuratus vergleichbar ist, ergibt sich hieraus eine außerordentlich gute Obereinstimmung und Bestätigung.

Um die vom Darm aufgenommene Sauerstoffmenge in ihrer Bedeutung richtig beurteilen zu können, darf man sie nicht einfach mit der gesamten Sauerstoffaufnahme vergleichen, sondern muß sie mit der dem Körperinnern zugeführten Sauerstoffmenge in Beziehung setzen, da ja der vom Darm aus aufgenommene Sauerstoff die $\mathrm{O}_{2}$-Versorgung des Körperinnern verbessern würde.

GIESE et al. (1966) haben bei Strongylocentrotus purpuratus die $\mathrm{O}_{2}$-Aufnahme der einzelnen Körperkomponenten gemessen und aus dem Verhältnis des Produktes aus $\mathrm{O}_{2}$-Aufnahme und Gewichtsanteil der Körperkomponenten (KörperkomponentenIndex) zum Gesamtkörpergewicht den relativen Anteil der Körperkomponenten an der gesamten $\mathrm{O}_{2}$-Aufnahme bestimmt. Thre Ergebnisse lassen sich, so wie sie sind, nicht auf Echinus esculentus übertragen, da dessen Körperkomponenten-Indizes infolge anderer Proportionen stark von denen des St. purpuratus abweichen. Es ist jedoch anzunehmen, daß die $\mathrm{O}_{2}$-Aufnahme der Körperkomponenten annähernd gleich bleibt. Setzt man dies voraus und ermittelt die Körperkomponenten-Indizes von E. esculentus so läßt sich auf die gleiche Weise wie es GIESE et al. getan haben auch für ihn der relative Anteil der einzelnen $\mathrm{Körperkomponenten} \mathrm{an} \mathrm{der} \mathrm{gesamten} \mathrm{O}_{2}$-Aufnalume abschätzen. Die entsprechenden Werte und Ergebnisse sind in Tabelle 4 zusammengefaßt.

Nimmt man nun eine $\mathrm{O}_{2}$-Aufnahme von $30 \mu \mathrm{O} \mathrm{O}_{2} / \mathrm{g} / \mathrm{h}$ an und errechnet für einen $160 \mathrm{~g}$ schweren E. esculentus den auf die im Körperinnern liegenden Organe (Gonaden, Darmtrakt, Metacoelflüssigkeit) entfallenden Anteil an der $\mathrm{O}_{2}$-Aufnahme, so ergeben sich die in Tabelle 4 (letzte Spalte) aufgeführten Werte. Gonaden, Metacoelflüssigkeit und Darm verbrauchen demnach zusammen $3142 \mu 1 \mathrm{O}_{2} / \mathrm{h}$, Darm und Metacoelflüssigkeit gemeinsam $613,93 \mu \mathrm{I} \mathrm{O} / \mathrm{h}$. Bei der normalen durchschnittlichen Trinkrate von ungefähr $0,5 \mathrm{ml} / \mathrm{h}$ für einen nicht erregten $E$. esculentus der oben angenommenen Größe, ist über den Darm eine maximale $\mathrm{O}_{2}$-Zufuhr von $3 \mu \mathrm{l} \mathrm{O} / \mathrm{h}$ möglich. Im Hinblick auf den gesamten Bedarf des Körperinneren erscheint diese Zufuhr als unbedeutend. 
Anders sieht die Sache jedoch aus, wenn es infolge einer Erregung durch äußere Reize zu einer Steigerung der Trinkfrequenz kommt. Ein E. esculentus von $160 \mathrm{~g}$ trinkt in erregtem Zustand bis zu $10,76 \mathrm{ml}$ Wasser pro Stunde, mit dem maximal $64,56 \mu \mathrm{O}_{2} / \mathrm{h}$ zugeführt werden können. Es sind dies mehr als $10 \%$ dessen, was Metacoelflüssigkeit und Darm gemeinsam aufnehmen und $2 \%$ des gesamten $\mathrm{O}_{2}$ Bedarfes des Körperinneren. Dieser Beitrag zur $\mathrm{O}_{2}$-Versorgung ist nicht gering $\mathrm{zu}$ achten, wenn man bedenkt, daß der Darm nach GIESE et al. in vitro von allen Organen (ausgenommen laichreife Testes) den höchsten $\mathrm{O}_{2}$-Verbrauch pro Zeit- und Gewichtseinheit hat. Zudem verursacht Erregung erhöhten $\mathrm{O}_{2}$-Verbrauch. Der daraus resultierende zusätzliche $\mathrm{O}_{2}$-Bedarf geht vermutlich in erster Linie von den Anhängen der Leibeswand aus. Füßchen, Pedizellarien und Stacheln zeigen erhöhte Aktivität. Es ist anzunehmen, daß der Anteil der Leibeswand am gesamten $\mathrm{O}_{2}$-Verbrauch steigt. Die $\mathrm{O}_{2}$-Versorgung des Körperinnern über die Füßchen wird sich etwas verringern, wenn die Füßchen höheren Eigenbedarf entwickeln und Stacheln sowie Pedizellarien in den interradialen Bereichen aus dem der Körperoberfläche zugeführten Atemwasser mehr entnehmen als im Ruhezustand.

Eine Regulierung der Atemwasserzufuhr durch Ventilationsmechanismen ist nicht vorhanden. Eine beschränkte Regulation, die eher einer Pufferwirkung gleichkommt, ergibt sich möglicherweise dadurch, daß die Schlagfrequenz der Coelothelwimpern zunimmt und eine raschere Umwälzung der Metacoelflüssigkeit herbeiführt, wodurch ein verbesserter $\mathrm{O}_{2}$-Transport zu den Organen zustande kommt, der letzte, ungenutzte $\mathrm{O}_{2}$-Reserven in der Coelomflüssigkeit mobilisiert.

Beobachtungen an eröffneten $E$. esculentus ergaben, daß mechanische Reizung nicht nur eine Erhöhung der Trinkfrequenz, sondern auch eine Steigerung der Darmperistaltik zur Folge hat. Erhöhte Peristaltik und Wimperschlag aber verbrauchen ihrerseits wieder mehr Sauerstoff. In dieser Situation könnte bei Seeigeln - vor allem mit zunehmender Größe - ein Teil ihres $\mathrm{O}_{2}$-Mehrbedarfs durch Steigerung der Trinkrate und $\mathrm{O}_{2}$-Aufnahme über den Darmtrakt gedeckt werden. Auch der Anstieg der Trinkrate mit abnehmender Anzahl der Füßchen pro Volumeneinheit - was praktisch eine Verschlechterung der $\mathrm{O}_{2}$-Versorgungslage bedeutet - spricht für eine Kompensation der $\mathrm{O}_{2}$-Versorgung auf enteralem Wege.

Um einen Größenvergleich für die in Erregung durch den Darm geschleuste Wassermenge zu bekommen, sei die Trinkrate von $E$. esculentus mit der Ventilationsrate der Wasserlungen von Holothuria forskali verglichen. Nach Newell \& CourTNeY nimmt $H$. forskali $60 \%$ ihres gesamten Sauerstoffbedarfes über die Wasserlungen mit einer Ventilationsrate von $48 \mathrm{ml} / \mathrm{h}$ auf; E. esculentus erreicht mit einer Trinkrate von $10,76 \mathrm{ml} / \mathrm{h}$ knapp ein Viertel davon.

Obwohl Seeigel keine speziellen respiratorischen Darmflächen aufweisen, bestehen doch von der Durchflußmenge, dem $\mathrm{O}_{2}$-Partialdruckgradienten, von der Dicke der Darmwand und der sie umspinnenden Blutgefäße her betrachtet, so gute Voraussetzungen, daß eine Aufbesserung der $\mathrm{O}_{2}$-Versorgung auf enteralem Wege im Erregungsfalle sehr wahrscheinlich ist. 


\section{Bedeutung für die Exkretion}

Wie die Versuchsergebnisse zeigen, scheidet E. esculentus in der Regel mehr Flüssigkeit durch den After aus, als er trinkt, was sich nur so erklären läßt, daß über die Körperoberfläche zusätzlich Wasser in das Leibesinnere eintritt. Dem steht - setzt man eine in beiden Richtungen gleiche Permeabilität voraus - sicherlich auch ein nicht zu vernachlässigender Wasseraustritt durch die Körperoberfläche gegenüber, der lösliche Stoffwechselendprodukte nach außen befördert, der jedoch in dieser Untersuchung nicht erfaßt werden konnte. Bei dem hier diskutierten Wasserstrom handelt es sich um einen Netto-Einwärtsfluß.

Die Füßchen und deren Ampullen sind, wie BinYon $(1961,1964)$ in seinen Arbeiten am Beispiel von Asterias rubens demonstriert, für Wasser extrem permeabel und da sich die Coelomräume (Meso- und Metacoel) osmotisch wie eine Einheit verhalten, sind auch im Körperinneren Wasserbewegungen von einer Leibeshöhle in die andere sehr gut möglich.

Auch die von Pequignat (1966) bei Seeigeln nachgewiesene KörperoberflächenVerdauung und -Resorption deutet auf einen einwärtsgerichteten Stofftransport hin, der vermutlich mit einem Wassertransport gekoppelt ist.

Ursache und Motor des Wassertransportes könnte ein Zusammenwirken von geringen osmotischen Differenzen zwischen Füßchenlumen und Außenwelt mit hydrostatischen, durch die Tätigkeit der Füßchen bedingten Druckunterschieden zwischen Füßchenampulle und Metacoel sein.

Zwar haben alle bisherigen Untersuchungen klar gezeigt, daß den Echinodermen die Fähigkeit zur Osmoregulation fehlt und sie im Rahmen der methodischen Genauigkeit meerwasserisoton sind. Dies schließt jedoch durch Stoffwechselvorgänge lokal (z. B. im Ambulacralsystem) leicht erhöhte osmotische Konzentrationen nicht aus, die einen Wasserfluß bewirken könnten.

Der Bestimmung der osmotischen Konzentration mit Hilfe der Gefrierpunkterniedrigung sind jedoch Grenzen gesetzt. Unter günstigsten Bedingungen läßt sich die Gefrierpunkterniedrigung auf $0,001^{\circ} \mathrm{C}$ genau ermitteln. Einer schon außerhalb der Fehlergrenze liegenden Gefrierpunkterniedrigung von $0,0009^{\circ} \mathrm{C}$ entspricht aber bereits ein osmotischer Druck von $10,8 \mathrm{~cm}$ Wassersäule, ein Druck, der z. B. halb so groß ist wie der, den die Füßchen bei maximaler Kontraktion überhaupt erzeugen können (FECHTER 1965). Es würden jedoch schon osmotische Drucke von wenigen Zentimetern genügen, um einen Wassertransport der in Frage stehenden Größenordnung zu bewirken.

Die in den Coelomflüssigkeiten vorhandenen Proteine (Holland et al. 1967) sind in ihrer Konzentration $z u$ gering und im Molekulargewicht zu hoch um einen ins Gewicht fallenden, kolloidosmotischen Gradienten erzeugen zu können. Anders dagegen verhält es sich mit der selektiven Anhäufung bestimmter Ionen. Nach den Untersuchungen von Bethe \& Berger (1931) sowie Robertson (1949) ist im Ambulacralsystem von $E$. esculentus ein Uberschuß von $\mathrm{K}^{+}-(5,7$ bzw. $2,6 \mathrm{mM})$ und $\mathrm{Ca}^{2+}$-Ionen $(4,6 \mathrm{mM})$ gegenüber dem umgebenden Meerwasser vorhanden, der anscheinend ständig aufrechterhalten wird. Dieser Ionenüberschuß könnte in zweifacher Hinsicht zu einem Wassertransport beitragen. Einmal wird bei der Aufnahme der Ionen aus dem Meerwasser 
Hydratationswasser miteingeschleppt. Welches Ausmaß dies annehmen kann, wird deutlich, wenn man bedenkt, daß im Mittel mit $1 \mathrm{mM} \mathrm{K}^{+} 68,4 \mu \mathrm{H}_{2} \mathrm{O}$ und mit $1 \mathrm{mM}$ $\mathrm{Ca}^{2^{+}}$gar 316,8 $\mu \mathrm{l} \mathrm{H}_{2} \mathrm{O}$ eingeschleust werden. Zum anderen repräsentiert der Ionen-

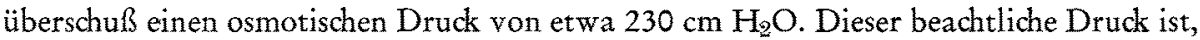
wie die Ausführungen zur Gefrierpunkterniedrigung zeigen, zumindest bis auf $10 \mathrm{~cm}$ $\mathrm{H}_{2} \mathrm{O}$ kompensiert. Es würde jedoch schon ein Druckgradient von $1 \mathrm{~cm} \mathrm{H} \mathrm{H}_{2} \mathrm{O}$, der einer erhöhten Ionenkonzentration von $0,044 \mathrm{mosmol}$ entspricht, genügen, um eine ausreichende Wasseraufnahme zu bewirken. Nimmt man dies an und setzt weiter voraus, daß der gesamte Netto-Wasserfluß über die Füßchenoberfläche erfolgt, so läßt sich die Permeabilität der Füßchen berechnen. Wenn a die Oberfläche eines Standardfüßchens (vgl. Fechter 1965), $n$ die Anzahl der Füßchen, $F$ der Netto-Wassereinstrom und $p$ der Druckgradient ist, ergibt sich die Wasserpermeabilität zu

$$
p=\frac{F}{a n p}
$$

Für den größten der untersuchten Seeigel, der maximal einen Netto-Wassereinfluß von $0,14(\mathrm{ml} / \mathrm{h}), 1890$ Füßchen und eine Füßchenstandardoberfläche von $0,312\left(\mathrm{~cm}^{2}\right)$ hat, ergibt sich bei $p=1 \mathrm{~cm} \mathrm{H} \mathrm{H}_{2} \mathrm{O}$ eine Permeabilität von $6,59 \cdot 10^{-8}\left(\mathrm{ml} / \mathrm{cm}^{2} / \mathrm{sec} / \mathrm{cm} \mathrm{H}_{2} \mathrm{O}\right)$. Dieser Wert stimmt größenordnungsmäßig sehr gut mit der Permeabiliät von $2,55 \cdot 10^{-8}$ $\left(\mathrm{ml} / \mathrm{cm}^{2} / \mathrm{sec} / \mathrm{cm} \mathrm{H}_{2} \mathrm{O}\right)$ überein, die BiNYoN (1964) für die gegenüber $E$. esculentus etwas derberen Füßchen von Asterias rubens ermittelte. Von der Permeabilität her betrachtet ist also ein osmotischer Gradient von $1 \mathrm{~cm} \mathrm{H}_{2} \mathrm{O}$ völlig ausreichend.

Besonders auffallend ist der hohe Wassereinstrom bei den kleinen Individuen. Für den kleinsten der untersuchten Seeigel $\left(a=0,194 \mathrm{~cm}^{2} ; F=0,509 \mathrm{ml} / \mathrm{h} ; n=1400\right)$ steigt die Permeabilität um das 7,8 fache auf $52,0 \cdot 10^{-8}\left(\mathrm{ml} / \mathrm{cm}^{2} / \mathrm{sec} / \mathrm{cm} \mathrm{H}_{2} \mathrm{O}\right)$. Dagegen stellte Binyon (1961) bei den kleinen Exemplaren von $A$. rubens nur eine Verdoppelung der Wasserpermeabilität fest. Nach den Untersuchungen BrNyons ist aber bei den kleinen Individuen der Ionenunterschied zum Meerwasser noch wesentlich ausgeprägter als bei den großen, so daß hier möglicherweise höhere $\left(>1 \mathrm{~cm} \mathrm{H} \mathrm{H}_{2} \mathrm{O}\right)$ osmotische Konzentrationen vorliegen, die einen dementsprechend vermehrten Wassereinstrom verursachen.

Das in die Füßchenlumina eingedrungene Wasser könnte durch reinen hydrostatischen Filtrationsdruck, wie er bei der Füßchenkontraktion auftritt, über die Ampullenwände in das Metacoel gepreßt werden. Die Füßchen von $E$. esculentus vermögen maximal einen Druck von $20 \mathrm{~cm} \mathrm{H}_{2} \mathrm{O}$ zu erzeugen. Da die Füßchenoberfläche etwa $2,7 \mathrm{mal}$ größer als die Oberfläche der Ampullen ist (FEcHTER 1965), müßten entweder die Permeabilität oder der wirksame Druckgradient bzw. das Produkt beider um mindestens diesen Faktor größer sein. Die im Vergleich zu den Füßchen viel geringere Wandstärke der Ampullen läßt auf eine gesteigerte Permeabilität derselben schließen, so daß ein wesentlich über $2 \mathrm{~cm} \mathrm{H}_{2} \mathrm{O}$ hinausgehender Druckgradient nicht erforderlich erscheint und über die Ampullen mehr abgepreßt wird als über die Füßchen, deren Permeabilität im kontrahierten Zustand zudem herabgesetzt sein dürfe.

Das ständige Wechselspiel von Kontraktion und Expansion der Füßchen, wie man es immer wieder beobachten kann, dient möglicherweise nicht nur der Sauerstoffversorgung des Körperinnern, sondern übernimmt zugleich auch die Rolle einer Wasserpumpe, die folgendermaßen arbeitet: In die expandierten, entspannten, nicht unter 
Binnendruck stehenden Füßßchen wird infolge eines geringen, einwärtsgerichteten osmotischen Gradienten, verbunden mit einer Ionenpumpe, Wasser in die Füßchenlumina geschleust. Bei der anschließenden Kontraktion der Füßchen wird das überschüssige, eingedrungene Wasser durch die Ampullenwände in das Metacoel abgepreßt. Danach werden die kontrahierten Füßchen durch Ampullenkontraktion wieder expandiert, die Ampullen entspannen sich, und der Kreislauf kann erneut beginnen.

Noch unveröffentlichte Untersuchungen zur Exkretion von Paracentrotus lividus (LAMARCK) haben ergeben, daß mindestens ein Drittel der stickstoffhaltigen Stoffwechselendprodukte über den Darm ausgeschieden wird. Auch van der Heyde (1923), DeLAUNAX (1931) und LEWIs (1967) schreiben dem Seeigeldarm eine exkretorische Funktion zu. Nach den hier vorliegenden Ergebnissen ist anzunehmen, daß diese Exkretion über den Darm mit einer aktiven Wasserausscheidung ohne Wasserrückresorption einhergeht, für die ja im marinen Milieu auch keine Notwendigkeit besteht. Das in den Darm ausgeschiedene, mit Stoffwechselendprodukten beladene Wasser wäre dann als eine Art Primärharn zu betrachten.

\section{ZUSAMMENFASSUNG}

1. Bei Echinus esculentus L. wurde die über den Mund aufgenommene und durch den After ausgeschiedene Wassermenge gemessen.

2. Die durch Trinken einverleibte Wassermenge nimmt allometrisch mit der Körpergröße zu. In erregtem Zustand ist eine Steigerung der Trinkrate um mehr als das 10 fache möglich.

3. Die durch den After ausgeschiedene Wassermenge ist größer als die über den Mund aufgenommene. Das bedeutet, daß über die Körperoberfläche zusätzlich Wasser absorbiert und durch den Darm wieder ausgeschieden wird. Diese zusätzliche Wasseraufnahme ist bei den kleinen Individuen besonders ausgeprägt.

4. Die Bedeutung des Trinkens, des Wassereinstromes über die Körperoberfläche und die Ausscheidung durch den Darm wird im Hinblick auf Atmung und Exkretion diskutiert. Dabei ergeben sich folgende Schlußfolgerungen: Das hastige Trinken in erregtem Zustand kann die Sauerstoffversorgung im Körperinneren verbessern, die Wasserausscheidung über den Darm die Exkretion von löslichen Stoffwechselendprodukten fördern.

5. Für die Wasseraufnahme durch die Körperoberfläche und den Weg des Wassers durch den Körper wird folgender Mechanismus vorgeschlagen und diskutiert: Infolge eines mit einer Ionenpumpe verbundenen $\mathrm{K}^{+}$- und $\mathrm{Ca}^{2+}$-Oberschusses in der Ambulacralflüssigkeit besteht ein geringer, einwärtsgerichteter osmotischer Gradient, der einen Wassereinstrom in die Lumina der expandierten Füßchen bewirkt. Durch Kontraktion der Füßchen wird das eingedrungene Wasser zum größten Teil über die Ampullenwände in das Metacoel gepreßt und vom Darm aktiv, zusammen mit Stoffwechselendprodukten, wieder ausgeschieden.

Danksagungen. Die Untersuchung wurde an der Biologischen Anstalt Helgoland durchgeführt. Dem Direktor der Anstalt, Herrn Prof. Dr. O. Kinne, danke ich herzlich für die gastfreundliche Aufnahme, ebenso Herrn A. HoLtmann für die aufmerksame Betreuung. 


\section{ZITIERTE LITERATUR}

Bethe, A. \& Berger, E., 1931. Variationen im Mineralbestand verschiedener Blutarten. Pflügers Arch. ges. Physiol. 227, 571-584.

Binyon, J., 1961. Salinity tolerance and permeability to water of the starfish Asterias rubens L. J. mar. biol. Ass. U.K. 41, 161-174.

- 1964. On the mode of functioning of the water vascular system of Asterias rubens L. J. mar. biol. Ass. U.K. 44, 577-588.

Delaunay, H., 1931. L'excrétion azotée des invertébrés. Biol. Rev. 6, 265-301.

Farmanfarmaian, A., 1966. The respiratory physiology of echinoderms. In: Physiology of Echinodermata. Hrsg. von R. A. Boolootian. Interscience Publ., New York, 245-265.

Fechter, H., 1965. Uber die Funktion der Madreporenplatte der Echinoidea. Z. vergl. Physiol. $51,227-257$.

FredericQ, L., 1911. La théorie de la diffusion suffit à expliquer les échanges gazeux de la respiration. Archs int. Physiol. 10, 391-413.

Giese, A. C., Farmanfarmaian, A., Hilden, S. \& Doezema, P., 1966. Respiration during the reproductive cycle in the sea urchin, Strongylocentrotus purpuratus. Biol. Bull. mar. biol. Lab., Woods Hole 130, 192-201.

HEYDE, H. C. VAN DER, 1923. Petites contributions à la physiologie comparée. IV. Sur l'excrétions chez les échinodermes. Archs néerl. Physiol. 8, 151-159.

Holland, L. Z., Grese, A. C. \& Phillips, J. H., 1967. Studies on the perivisceral coelomic fluid protein concentration during seasonal and nutritional changes in the purple sea urchin. Comp. Biochem. Physiol. 21, 361-371.

Johansen, K. \& VADAS, R. L., 1967. Oxygen uptake and responses to respiratory stress in sea urchins. Biol. Bull. mar. biol. Lab., Woods Hole 132, 16-22.

Koller, G. \& MeYer, H., 1933. Versuche uiber die Atmung der Echinodermen. Biol. Zbl. 53, 655-661.

KRÜGER, F, 1932. Versuche über die Wasserbewegung durch die Madreporenplatte von Echinus esculentus. Z. vergl. Physiol. 18, 157-173.

LEWIS, J. B., 1967. Nitrogenous excretion in the tropical sea urchin Diadema antillarum Phimippi. Biol. Bull. mar. biol. Lab., Woods Hole 132, 34-37.

Newell, R. C. \& Courtney, W. A. M., 1965. Respiratory movements in Holotburia forskali DELle Chiaje. J. exp. Biol. 42, 45-57.

Pequignat, E., 1966. "Skin digestion" and epidermal absorption in irregular and regular urchins and their probable relation to the outflow of spherule-coelomocytes. Nature, Lond. 210, 397-399.

Perrier, E., 1875. Recherches sur l'appareil circulatoires des oursins. Archs Zool. exp. gén. 4, $605-643$.

Robertson, J. D., 1949. Ionic regulation in some marine invertebrates. J. exp. Biol. 26, $182-200$.

SteEN, J. B., 1965. Comparative aspects of the respiratory gas exchange of sea urchins. Acta physiol. scand. 63, 164-170.

STотт, F. C., 1955. The food canal of the sea-urchin Echinus esculentus L. and its functions. Proc. zool. Soc. Lond. 125, 63-86.

Anschrift des Autors: Dr. H. Fechter

Zoologische Staatssammlung München

8 München 19

Maria-Ward-Straße 1b

Bundesrepublik Deutschland 